\title{
Cosolvent Flushing for the Remediation of PAHs from Former Manufactured Gas Plants
}

\author{
P.S. Birak, A.P. Newman, S.D. Richardson, S.C. Hauswirth, J.A. Pedit, M.D. Aitken, and C.T. \\ Miller \\ Department of Environmental Sciences and Engineering, University of North Carolina, Chapel \\ Hill, North Carolina 27599-7431, USA \\ P.S.Birak: pamela_birak@unc.edu; A.P.Newman: newman.arne@gmail.com; S.D. Richardson: richardsonS@solutions- \\ ies.com; S.C. Hauswirth: shaus@email.unc.edu; J.A. Pedit: pedit@email.unc.ed; M.D. Aitken: aitken@email.unc.edu
}

\begin{abstract}
Cosolvent flushing is a technique that has been proposed for the removal of hydrophobic organic contaminants in the subsurface. Cosolvents have been shown to dramatically increase the solubility of such compounds compared to the aqueous solubility; however, limited data are available on the effectiveness of cosolvents for field-contaminated media. In this work, we examine cosolvent flushing for the removal of polycyclic aromatic hydrocarbons (PAHs) in soil from a former manufactured gas plant (FMGP). Batch studies confirmed that the relationship between the soil-cosolvent partitioning coefficient $\left(K_{i}\right)$ and the volume fraction of cosolvent $\left(f_{c}\right)$ followed a standard log-linear equation. Using methanol at an $f_{c}$ of 0.95 , column studies were conducted at varying length scales, ranging from 11.9 to $110 \mathrm{~cm}$. Removal of PAH compounds was determined as a function of pore volumes (PVs) of cosolvent flushed. Despite using a high $f_{c}$, rate and chromatographic effects were observed in all the columns. PAH effluent concentrations were modeled using a common two-site sorption model. Model fits were improved by using $\mathrm{MeOH}$ breakthrough curves to determine fitted dispersion coefficients. Fitted mass-transfer rates were two to three orders of magnitude lower than predicted values based on published data using artificially contaminated sands.
\end{abstract}

\section{Keywords}

Sorption; Dissolution; Polycyclic aromatic hydrocarbons; Coal tar; Rate effects; Fieldcontaminated soils; Methanol; FMGP

\section{Introduction}

The manufacture of gas from coal and oil was widespread throughout the U.S. and Europe during the 1800s to mid 1900s. In the U.S. alone, there are an estimated 33,000 to 50,000 former manufactured gas plants (FMGPs) and related sites, which are suspected of having soil and groundwater contamination. The most common contaminant is tar, a by-product of the manufacturing process (U.S. EPA, 2004).

(C) 2011 Elsevier B.V. All rights reserved.

*Corresponding author: casey_miller@unc.edu (C.T. Miller).

Publisher's Disclaimer: This is a PDF file of an unedited manuscript that has been accepted for publication. As a service to our customers we are providing this early version of the manuscript. The manuscript will undergo copyediting, typesetting, and review of the resulting proof before it is published in its final citable form. Please note that during the production process errors may be discovered which could affect the content, and all legal disclaimers that apply to the journal pertain. 
FMGP tars are complex mixtures of mostly organic compounds. Though the exact chemical composition is still unknown and varies from site to site, tars contain a large percentage of PAHs, including several carcinogens (U.S. EPA, 1993). In most tars, naphthalene (NAP), a two-ring PAH, is the most abundant individual compound, accounting for as much as $15 \%$ of tar mass (Birak and Miller, 2009). As the number of rings increases, concentrations in tar generally decrease. For example, benzo(a)pyrene $(\mathrm{BaP})$, a five-ring $\mathrm{PAH}$, is usually less than $1 \%$ of tar mass (Birak and Miller, 2009).

In the subsurface, tars are long-term sources of soil and groundwater contamination. When released to soil, tars exhibit complex migration patterns that are dependent on local geology. If tars migrate downward and encounter the water table, they may sink further as they are dense, non-aqueous phase liquids (DNAPLs). Tar-contaminated systems can become oilwet, resulting in soil and sand grains being coated with tar (Barranco and Dawson, 1999; Powers et al., 1996). PAHs dissolve from the DNAPL phase into bulk groundwater slowly because of low aqueous solubilities (Luthy et al., 1994).

Tar composition and properties are altered over time as tars age. Lower molecular weight (MW) compounds, such as NAP, are depleted first, primarily due to dissolution (Luthy et al., 1994). Over time, the mole fractions of higher MW PAHs increase, along with their aqueous-phase concentrations (Peters et al., 1999). This results in a chromatographic effect occurring down-gradient of tar-contaminated zones, as compounds are eluted at varying time-scales. Over time, dissolution can result in tars becoming increasingly viscous, and they may ultimately become solids or semi-solids (Peters et al., 1997).

Cosolvent flushing is a technique that has been proposed for the removal of hydrophobic organic contaminants in the subsurface. Cosolvents can markedly increase the solubility of compounds, such as PAHs. This relationship has been found to follow a log-linear equation, such that

$$
\log C_{i}^{s a t}=\log C_{i w}^{s a t}+\sigma_{i} f_{c}
$$

where $C_{i}^{\text {sat }}$ is the solubility in a cosolvent for species $i, C_{i w}^{\text {sat }}$ is the solubility in water, $\sigma_{i}$ is the cosolvency power, and $f_{c}$ is the cosolvent volume fraction (Yalkowsky, 1981). In soils, cosolvents reduce the equilibrium partition coefficient, such that

$$
\log K_{i}=\log K_{i w}-\alpha \sigma_{i} f_{c}
$$

where $K_{i}$ is equilibrium partition coefficient between the cosolvent and soil $\left(\mathrm{L}^{3} / \mathrm{M}\right), K_{i w}$ is the equilibrium partitioning coefficient between water and soil $\left(\mathrm{L}^{3} / \mathrm{M}\right)$, and $\alpha$ is an empirical constant that accounts for sorbent-alcohol interactions (Rao et al., 1990; Wood et al., 1990).

Cosolvents have also been found to reduce mass transfer rates. Brusseau et al. (1991) and Bouchard (1998) conducted column experiments to examine this relationship using breakthrough curves of PAHs in the presence of methanol $(\mathrm{MeOH})$ at varying $f_{c}$. The initial condition was a column packed with uncontaminated Eustis sand saturated with a $\mathrm{MeOH}$ cosolvent solution. Brusseau et al. (1991) and Bouchard (1998) pumped solutions with a known PAH concentration and the same $f_{c}$ already present in the column. Effluent concentrations were simulated using a two-site sorption model where one fraction of the mass was assumed to remain in equilibrium and the remaining fraction was rate-limited, such that 


$$
\begin{gathered}
R_{i} \frac{\partial C_{i}}{\partial t}=-v \frac{\partial C_{i}}{\partial x}+D \frac{\partial^{2} C_{i}}{\partial x^{2}}-\frac{\rho_{b}}{\theta} \frac{\partial S_{2 i}}{\partial t} \\
\frac{\partial S_{2 i}}{\partial t}=k_{i}\left[\left(1-F_{i}\right) K_{i} C_{i}-S_{2 i}\right] \\
R_{i}=\frac{\rho_{b}}{\theta} K_{i} F_{i}+1 \\
S_{i}=S_{1 i}+S_{2 i}
\end{gathered}
$$

where $R_{i}$ is the retardation factor, $C_{i}$ is the concentration in the aqueous phase $\left(\mathrm{M} / \mathrm{L}^{3}\right), S_{i}$ is the solute mass fraction in soil (M/M), $S_{1 i}$ is a solute-solid mass fraction assumed to be in equilibrium (M/M), $S_{2 i}$ is a solute-solid mass fraction that is rate-limited $(\mathrm{M} / \mathrm{M}), v$ is the linear pore velocity $(\mathrm{L} / \mathrm{T}), D$ is the hydrodynamic dispersion coefficient $\left(\mathrm{L}^{2} / \mathrm{T}\right), \rho_{b}$ is the bulk density $\left(\mathrm{M} / \mathrm{L}^{3}\right), \theta$ is the porosity $\left(\mathrm{L}^{3} / \mathrm{L}^{3}\right), k_{i}$ is the mass transfer rate $(1 / \mathrm{T})$, and $F_{i}$ is the mass fraction of the solid phase assumed to always be in equilibrium with the aqueous phase.

The model was fit to determine values for $k_{i}$ and $F_{i}$. For individual PAHs, correlations were found between $k_{i}$ and $f_{c}$, such that

$$
\log k_{i}=\log k_{i w}-\varphi_{i} f_{c}
$$

where $k_{i w}$ is the mass transfer rate in a pure aqueous phase $(1 / \mathrm{T})$, and $\varphi_{i}$ is a constant (Brusseau et al., 1991). Data from multiple chemicals were used to develop a more general relationship between the rate and the partitioning coefficient

$$
\log k_{i}=\tau \log K_{i}+b
$$

Brusseau et al. (1991) reported a $\tau$ of -0.61 and $b$ of 0.79 based on data for NAP and phenanthrene (PHE), where $k_{i}$ is in $\mathrm{hrs}^{-1}$. Bouchard (1998) included additional PAHs and reported a value for $\tau$ of -0.91 and for $b$ of 0.47. In addition, Brusseau et al. (1991) found that $F_{i}$ was lower as the cosolvent fraction increased, but was unable to develop a relationship to predict $F_{i}$ based on other parameters.

Augustijn et al. (1994) used the two-site model to simulate column desorption experiments. In these experiments, the initial condition was an aqueous solution containing either NAP or PHE in equilibrium with Eustis sand. Cosolvent solutions were then pumped through the columns and the effluent concentrations measured over time. They modeled the system using the rate constants from Brusseau et al. (1991) and found that model predictions generally agreed with experimental data. 
A limitation of these previous modeling studies is that they all relied on single-solute systems of artificially contaminated media. Thus, the effects of aging and the complexity of contaminant mixtures were not considered. In terms of PAHs, previous studies have also tended to use compounds that have relatively low MW when compared to the entire suite of 16 U.S. EPA priority pollutant PAHs that are of concern from a risk perspective. These studies were also all conducted at the same length scale of approximately $5 \mathrm{~cm}$. It has been well documented that remediation efficiency diminishes with length scale (Soga et al., 2004).

Few studies have been conducted to examine the removal of PAHs from field soils. Using an FMGP soil, Chen et al. (2005) conducted column experiments and flushed $10 \mathrm{PVs}$ of $\mathrm{MeOH}$ or ethanol (EtOH) at an $f_{c}$ of 0.85 in $5.5-\mathrm{cm}$ columns. Percent removal was lowest for the high MW compounds. For example, the percent removal for NAP was $67.9 \%$ using $\mathrm{MeOH}$ and $69.9 \%$ using EtOH. For BaP, removal was $42.3 \%$ using $\mathrm{MeOH}$ and $27.3 \%$ using $\mathrm{EtOH}$. Since these results were not modeled, mass transfer rates were not reported. Kilbane (1998) conducted batch experiments with an aged FMGP soil and removal rates were near $100 \%$ using pure ethanol, suggesting that it may be possible to achieve higher removal percentages by using a higher $f_{c}$.

The overall goal of this work was to evaluate the use of alcohol flushing for the remediation of field-contaminated and aged soils typical of FMGPs. The specific objectives were: (1) to investigate the remediation of aged, field-contaminated solid materials from FMGPs using cosolvent flushing; (2) to observe the effects of system hydrodynamic characteristics on contaminant mass removal efficiency; (3) to evaluate the presence and importance of mass transfer limitations as a function of solute properties; and (4) to assess the adequacy of a commonly posited model for field-contaminated systems.

\section{Materials and Methods}

\subsection{Field Samples}

Field samples were collected from a FMGP site in Salisbury, North Carolina. The highest PAH concentrations were detected in boreholes located between the former retort house and tar well. Soil samples were collected from this region at a depth of approximately $1.2 \mathrm{~m}$ and stored in sealed buckets at $4^{\circ} \mathrm{C}$ prior to processing. No free liquid-phase tar was observed in the field soil.

The field soil was screened through a 10-mm wire screen to break up large aggregates and remove rocks, brick fragments, and other debris. The screened soil was thoroughly mixed to create a homogeneous soil stock. The homogenized soil was then mixed with sterile 40/50grade silica sand (Accusand, Unimin Corporation, Le Sueur, MN) at a 1:1 mass ratio to allow flow through packed columns with a sufficiently small pressure gradient.

Properties of soil mixture are given in Table 1. The soil organic carbon (OC) totaled 8.3\%; however, the total extractable organic matter (TEOM) was $0.64 \%$. The TEOM was determined gravimetrically from the soil extracts as described in §2.2.2. It is important to note that these data are based on a few samples; however, the difference between the \%OC and the \%TEOM is consistent with other analyses of aged tar-contaminated soil.

Specifically, most of the organic matter in similar soils has been identified as a heavy nonextractable fraction, believed to be the result of tar aging (Haeseler et al., 1999).

\subsection{Analytical Methods}

2.2.1 PAH Analysis-PAHs were isolated from the soil mixture using a two-step solvent extraction procedure. Five-gram (wet wt.) aliquots of the soil mixture were extracted using 
$20 \mathrm{ml}$ of dichloromethane-acetone $(1: 1, \mathrm{v} / \mathrm{v})$. Sodium sulfate $(6-7 \mathrm{~g})$ was added to each extraction vial to absorb residual water, and three to six 5-mm diameter glass beads were added to each vial to improve soil-solvent mixing. Anthracene- $\mathrm{d}_{10}$ was used as an internal standard (Restek, Bellefonte, PA) to evaluate analytical recovery. Vials were sealed with Teflon-lined screw caps, vigorously shaken for $24 \mathrm{hr}$, and centrifuged for $15 \mathrm{~min}$ at 3,500 $\mathrm{rpm}(1,800 \mathrm{G})$. The supernatant was removed and the extraction was repeated. The extracts from each step were combined and stored in the dark at $4^{\circ} \mathrm{C}$ prior to analysis.

Sample extracts were analyzed using a Waters (Milford, MA) HPLC equipped with a 10-cm, 3- $\mu \mathrm{m}$ particle size Supelcosil ${ }^{\mathrm{TM}}$ LC-PAH column (Sigma-Aldrich, St. Louis, MO), a fluorescence detector, and HPLC grade solvents. Over the course of the column experiments, two HPLC methods were used as a result of improvements to the analysis. Method I was a 20-min instrument method with a starting mobile phase of acetonitrile-water $(60: 40, \mathrm{v} / \mathrm{v})$. Method II was a 45-min instrument method with a starting mobile phase of acetonitrile-water $(55: 45, \mathrm{v} / \mathrm{v})$, which improved peak separation. In both methods, acetonitrile was increased linearly to $100 \%$ over the course of each run. External calibration standards were prepared from a PAH analyte mixture (SPEX Certiprep CLPS-B, Metuchen, NJ). Of the 16 U.S. EPA priority pollutant PAHs, two PAHs were not quantified, acenaphthylene, which does not fluoresce, and indeno[1,2,3-cd]pyrene, which had concentrations too low to detect.

2.2.2 TEOM Analysis-Field soil extracts were fractionated to better understand the overall composition of the TEOM. First, soil was screened through a 2-mm wire mesh and extracted with dichloromethane and acetone, as described above. To precipitate the asphaltene fraction, n-pentane was added to the extracts at a ratio of $40 \mathrm{~mL}$ per $\mathrm{mL}$ of extract. Samples were vacuum-filtered through $0.2-\mu \mathrm{m}$ pore-size nylon filters. The precipitate was dried under nitrogen and weighed. To determine what is referred to as the "neutral" fraction, the filtrate was passed through a solid-phase extraction column containing $2 \mathrm{~g}$ of Florisil, dried under nitrogen and weighed. Finally, the sample mass retained on the column was eluted using toluene-acetone $(1: 1, \mathrm{v} / \mathrm{v})$, which was also dried under nitrogen. This fraction is referred to as the "polar" fraction. A Hewlett-Packard 5890 gas chromatograph, coupled with a Hewlett-Packard 5971 quadrapole mass spectrum detector was used for the semi-quantitative analysis of the neutral fraction.

2.2.3 Density Measurements-Density was measured in batch extracts and column effluent samples using an Anton Paar density meter (Model DMA 48) calibrated at $22^{\circ} \mathrm{C}$, the average temperature in the laboratory. These density measurements were converted to methanol $f_{c}$ using empirical data. In $\mathrm{MeOH}$ :water solutions, converting between density and volume fraction is complicated by the fact that volume is not conserved. As published data were not found for $22^{\circ} \mathrm{C}$, density was measured in solutions of known MeOH $f_{c}$ (Fisher, ACS Certified) and distilled-deionized water (DD) with mass fractions from zero to one. For each solution, the $f_{c}$ was calculated from the $\mathrm{MeOH}$ mass fraction, $\omega$, and measured solution density, $\rho$, as

$$
f_{c}=1.266 \omega \rho
$$

where $\rho$ is expressed with units of $\mathrm{g} / \mathrm{ml}$. These data are provided in Table 2 and were in excellent agreement with data published at other temperatures (Gonzalez et al., 2007). A piecewise cubic Hermite interpolating polynomial was generated from the data using MATLAB's pchip function. Since column studies used simulated groundwater, we confirmed that this relationship held for cosolvent mixtures using simulated groundwater as 
well. For samples with unknown $\mathrm{MeOH}$ concentration, the polynomial was evaluated to convert measured $\rho$ to $f_{c}$.

\subsection{Batch Experiments}

Batch experiments were conducted to determine the relationship between $K_{i}$ and $f_{c}$. The batch experiments were performed using $f_{c}$ values of 0 to 1 in increments of 0.1 , and at an $f_{c}$ of 0.95 . Batch experiments were conducted in triplicate for each $f_{c}$. An approximately $3 \mathrm{~g}$ portion of the soil mixture was added to $20 \mathrm{~mL}$ of $\mathrm{MeOH}$ solution in a centrifuge vial and shaken for $48 \mathrm{hr}$. Vials were centrifuged for $15 \mathrm{~min}$ at 3,500 rpm $(1,800 \mathrm{G})$. The cosolvent was decanted and analyzed for PAHs as described in $\$ 2.2 .1$ to determine the concentration of PAHs in the cosolvent $\left(C_{i}^{e q}\right)$. The total starting solute masses in the soil mixture $\left(M_{0 i}\right)$ were determined by extracting the remaining mass of PAH as described in $\$ 2.2$ and adding to the mass removed from the cosolvent. The $K_{i}$ was calculated as follows

$$
K_{i}=\frac{M_{0 i}-C_{i}^{e q} V}{C_{i}^{e q} M_{s}}
$$

where $M_{s}$ is the dry mass of the soil mixture and $V$ is the volume of the cosolvent solution.

\subsection{Column Experiments}

For each column, the same general experimental procedure was followed: (1) wet-packing with the soil mixture, (2) determination of the dispersion coefficient using a tracer test, (3) flushing with a $\mathrm{MeOH}$ solution at an $f_{c}$ of 0.95 under density stable conditions, (4) collection and analysis of effluent samples, and (5) analysis of the soil after flushing. The design parameters of the columns are given in Table 3. (See Table 1 for properties of the soil mixture and Table 5 for concentrations of PAHs in soil mixture.)

For $\mathrm{C} 1, \mathrm{C} 3$, and $\mathrm{C} 4$, a glass column was equipped with stainless steel end caps and tubing. The columns were wet packed over a $10 \mathrm{~g}$ bed of 30/40 Accusand. The soil mixture was then introduced in $0.5 \mathrm{~cm}$ lifts, each lift followed by either slight vibration (using a pneumatic vibrator) or tapping of the column walls. Gentle stirring was also used to remove entrained air bubbles and to ensure amalgamation of the lift layers. In C4 only, the column was also topped with an additional layer of Accusand. This layer was added so the column could be flipped $180^{\circ}$ post colsolvent flushing and prior to flushing with water; thus, we were able to maintain a density stable front without reversing the direction of flow. A syringe pump was used to maintain flow through the columns (Harvard Apparatus; Model 44).

For C2, a 110-cm column was constructed out of 10.2-cm outer diameter stainless steel pipe. Three evenly-spaced sampling ports were located at 30,55 , and $80 \mathrm{~cm}$ below the column top. The column was wet-packed over a 5-cm bed of oven-dried, autoclaved 30/40 Accusand sand. The soil mixture was introduced in 3 to $5 \mathrm{~cm}$ lifts followed by vibration of the column walls (using a pneumatic vibrator) and gentle stirring. A stainless steel single-piston metering pump (Eldex Laboratories, Inc., Napa, CA) was used to maintain flow. This column was one of several constructed for studying biological and chemical treatment of soil. Further detail on the column design can be found in Richardson et al. (2011).

Prior to alcohol flushing, the columns were equilibrated with a simulated groundwater solution, based on historical groundwater ion concentrations near Salisbury, North Carolina (Harden et al., 2009). This solution was prepared by adding $1.83 \mathrm{~g} \mathrm{CaCl}_{2} \cdot 2 \mathrm{H}_{2} 0,1.01 \mathrm{~g}$ 
$\mathrm{MgSO}_{4} \cdot 7 \mathrm{H}_{2} 0,2.19 \mathrm{~g} \mathrm{NaHCO} 3,1 \mathrm{~mL}$ of $8.77 \mathrm{~g} / \mathrm{L} \mathrm{KCl}$ solution, and $1 \mathrm{ml}$ of $1 \mathrm{~N} \mathrm{H}_{2} \mathrm{SO}_{4}$ to $20 \mathrm{~L}$ of sterile-filtered DI water (ionic strength $=4.5 \mathrm{E}-3$ ). The solution was also de-aired to help remove entrained air bubbles. For $\mathrm{C} 2$ only, the column operated continuously for approximately 11 months prior to cosolvent flushing. Soil samples were analyzed for PAHs immediately before cosolvent flushing to assess the loss of PAH mass during the equilibration period.

2.4.1 Tracer Tests-Tritium was used as a conservative tracer to determine the hydrodynamic dispersion coefficient, $D$, by fitting breakthrough curves to the advectiondispersion equation. A $16 \mathrm{nCi} / \mathrm{mL}$ tracer solution was prepared by combining a stock solution of tritiated water with simulated groundwater. Effluent samples were collected in glass scintillation vials, combined with a scintillation cocktail (Fisher Scientific SX23-5), and analyzed on a Packard Tri-Carb Liquid Scintillation Analyzer (Meriden, CT; Model 1900 TR).

In C2, approximately $0.5 \mathrm{PVs}$ of tritiated groundwater was pumped into the column, followed by continuous flushing with non-tritiated groundwater. For $\mathrm{C} 2$, the tracer test was run soon after the column was prepared, prior to the equilibration period and alcohol flushing. In $\mathrm{C} 3$ and $\mathrm{C} 4$, the tritiated groundwater was pumped through the columns until complete breakthrough was observed (approximately two PVs), followed by continuous pumping of non-tritiated groundwater. A tracer test was not run on column $\mathrm{C} 1$, though we would expect the dispersion coefficient to be similar to column C3.

2.4.2 Cosolvent Flushing-In Table 4, the flushing strategy for each column is provided. In three of the columns, flow was halted during cosolvent flushing to test for rate effects. In $\mathrm{C} 3$ and $\mathrm{C} 4$, the time for the stop flow was increased after rate effects were observed in $\mathrm{C} 1$. During flushing, effluent samples were collected in glass vials equipped with PTFE-lined screw caps. In the smaller scale experiments (C1, C3, C4), effluent was continuously collected. Sample vials were initially changed hourly during $\mathrm{C} 1$ and $\mathrm{C} 3$, and every half-hour during $\mathrm{C} 4$. In the largest column (C2), the samples were collected intermittently on an hourly basis, with $10 \mathrm{ml}$ of effluent collected during each sampling event. In all the columns, the time between samples was increased as the change in concentrations decreased.

Effluent samples were collected in vials pre-filled with acetonitrile for all the columns except $\mathrm{C} 4$, for which samples were collected in $\mathrm{MeOH}$. Effluent samples were analyzed for PAHs using HPLC as described in \$2.2.1. Most samples required further dilution prior to HPLC analysis. Because the concentrations of individual PAHs increased rapidly during cosolvent flushing, it was necessary to change the dilution factor between samples, periodically causing some PAHs to fall near or below the quantification limit. In $\mathrm{C} 4$, two or three different dilution factors were used for each sample to ensure concentrations above the detection limit. Density was also measured in $\mathrm{C} 4$. Since the amount of $\mathrm{MeOH}$ pre-added to each vial was known, the estimated $\mathrm{MeOH}$ concentration was corrected for the pre-added $\mathrm{MeOH}$.

After cosolvent flushing, the columns were flushed with simulated groundwater for two to three PVs. In C4, the water flush began immediately after the stop flow and the column was rotated $180^{\circ}$, such that the flow direction was maintained along with a density stable front. For this column only, effluent samples were collected during the water flush and a complete breakthrough curve could be determined for $\mathrm{MeOH}$.

2.4.3 Post-flushing Soil Analysis—The column soil was extracted as described in §2.2. For the experiments conducted in the glass columns (C1, C3, C4), the soil was removed and 
divided into four layers along the length of the column. Each section was homogenized and a portion was used for moisture content analysis. For C2, soil samples were taken immediately before and after alcohol flushing from each of the three soil sampling ports. A portion of these samples was also used to determine the moisture content. All samples were analyzed in triplicate for PAHs.

In the glass column experiments, the final mean soil concentration was calculated as a weighted average of the concentration in each layer. In C2, the final mean soil concentrations were calculated by taking the mean of the concentrations from each sampling port. The initial concentrations were estimated by summing the mass remaining postflushing with the mass removed during flushing, which was determined by integrating the effluent concentrations with respect to the volume flushed using the trapezoidal rule.

2.4.4 Modeling of Cosolvent Flushing-A code was written in Matlab to solve the two-site model (Eqn 3-6). Advection, dispersion and desorption were solved numerically using a finite-difference alternating-split-operator approach. Advection was solved explicitly using MUSCL as described in Farthing and Miller (2000) and dispersion was solved implicitly using a centered-difference formula. For the cosolvent flushing, desorption was solved for each node using an ODE solver (Matlab, ode15s). Experimental data were fit using the trust-region-reflective lease-squares algorithm (Matlab, lsqcurvefit).

\section{Results}

\subsection{Soil Analysis}

The PAH mass in the soil mixture is presented in Table 5. The wide range of values demonstrates the heterogeneity of the mixtures and the distribution of PAHs suggests that the tar is highly weathered. In unweathered tar samples, NAP is typically the most prevalent PAH (Birak and Miller, 2009). For this soil, PHE was the most prevalent PAH. The relatively low amount of NAP is consistent with other analyses of highly weathered tarcontaminated soils (Liu et al., 2009), although the soil processing methods in the current study could have also lead to additional losses of NAP by volatilization.

In addition to quantifying individual compounds, soil extracts (i.e., TEOM) were fractionated into asphaltenes, polar compounds, and neutral compounds. These data are presented in Table 6 . This analysis revealed that $46 \%$ of the TEOM was in either the asphaltene or polar fraction. The neutral fraction was further analyzed by GC-MS. The GCMS data were used to conduct a semi-quantitative analysis of the neutral fraction based on relative peak area. Based on this analysis, the U.S. EPA priority pollutant PAHs contributed to $24 \%$ of the total peak area. The most prominent group of compounds were the methylphenanthrenes, which accounted for $40 \%$ of the total peak area. The remaining peak area consisted of a large number of much smaller peaks that were typically $1 \%$ or less of the total peak area.

\subsection{Batch Experiments}

The batch data were used to determine the cosolvent partition coefficient, $K_{i}$, for $f_{c}$ ranging from 0 to 1 . In Fig $1, \log K_{i}$ is plotted as a function of $f_{c}$ for PHE and BaP. As shown in Fig 1 , the $K_{i}$ sometimes spanned as much as an order of magnitude for a given $f_{c}$, while the high MW PAHs were often below the quantification limit for the low $f_{c}$ batch tests. Regardless, the data were well fit by the log-linear model (Eq. 2), for which the coefficients and corresponding $\mathrm{R}^{2}$ values are provided in Table 7 . Limited data are available to compare to previous studies; however, $\alpha \sigma$ values for NAP and PHE compared well to $\sigma$ values published by Bouchard (1998) for artificially contaminated sands of 2.52 for NAP and 4.69 for PHE. 
Bouchard (1998) also reported values for $K_{i w}$ of 0.58 for NAP and 2.33 for PHE. These are considerably lower than the values determined for the soil mixture, which makes sense given the aging of the field contaminated media.

\subsection{Tracer Tests}

A standard advective-dispersive model was fit to the tracer test data. Results are given in Table 3 for all the columns and plots are given in Fig $2 \mathrm{a}$ and $3 \mathrm{a}$ for $\mathrm{C} 2$ and $\mathrm{C} 4$. All the data were well fit by the advective-dispersive model.

The tracer data were used to predict the $\mathrm{MeOH} f_{c}$ in the cosolvent flushing experiment in $\mathrm{C} 2$. In Fig $2 \mathrm{~b}$, the modeled $f_{c}$ is compared to estimates based on the mass and volume of each sample. This is a relatively imprecise method but does provide some indication that the $\mathrm{MeOH}$ concentrations are reasonably estimated using the dispersivity from the tracer tests, although there does appear to be slightly sharper breakthrough of $\mathrm{MeOH}$ as compared to the conservative tracer.

For $\mathrm{C} 4$, the density was measured in the effluent samples, providing a more precise estimate of $f_{c}$. In this case, the dispersion data from the conservative tracer did not provide a good fit to these data. In addition, the breakthrough-curve for $\mathrm{MeOH}$ was not symmetric; thus, the up and down portion were fit separately and are given in Fig $3 \mathrm{~b}$. Though fitting the up and down portion of the curve was an improvement, the $\mathrm{MeOH}$ data still deviated from the advection-dispersion model.

\subsection{Column Flushing Experiments}

Cosolvent flushing was found to significantly reduce the mass of PAHs in all the column experiments. Fig 4 compares $S_{i}$ pre- and post-flushing in $\mathrm{C} 1$ and $\mathrm{C} 2$. Values are shown for the 10 PAHs that were consistently quantifiable during these experiments. The values of $S_{i}$ pre-flushing are estimated as described in $\$ 2.4 .3$ and are in reasonable agreement with measurements from the soil mixture. We also did not find any significant difference in the measured values immediately before flushing. The values of $S_{i}$ post-flushing are averages measured along the extent of the column. Based on the 10 PAHs in Fig 4, the PAH mass was reduced $93 \%$ in $\mathrm{C} 1$ and $95 \%$ in $\mathrm{C} 2$.

Although the total PAH mass removed was similar between $\mathrm{C} 1$ and $\mathrm{C} 2$, achieving the same amount of mass removal in $\mathrm{C} 2$ required more PVs of cosolvent flushed as compared to $\mathrm{C} 1$. In Fig 5, the cumulative fraction of mass removed is plotted for the first $10 \mathrm{PVs}$ flushed. The number of PVs required to remove $50 \%$ of the PAH mass was 1.8 in $\mathrm{C} 1$ and 3.9 in $\mathrm{C} 2$, while $90 \%$ removal required 6.5 in $\mathrm{C} 1$ and 9.5 in $\mathrm{C} 2$.

We also found that individual compounds eluted over different time scales. In Fig 5, the cumulative mass removed for individual compounds is plotted. These curves are grouped by the number of rings for a given PAH. For the most part, the three, four, and five-ring PAHs eluted in order of decreasing solubility. Interestingly, NAP eluted last, despite having the highest solublity. Also, NAP was the only compound to show a slight increase in concentration at the stop flow in $\mathrm{C} 1$.

In $\mathrm{C} 3$ and $\mathrm{C} 4$, longer stop flows were allowed and rate effects were more clearly observed. In these experiments, all the PAHs in Table 5 were quantifiable. The total PAH mass was reduced $89 \%$ in $\mathrm{C} 3$ and $81 \%$ in $\mathrm{C} 4$. In Fig 6, the cumulative mass removal is plotted for $\mathrm{C} 3$ and C4. During the stop in flow, NAP had the most significant increase in relative mass removed followed by the three and four-ring PAHs. The five and six-ring PAHs had relatively small increases in concentrations that are not visible on this scale. 


\subsection{Modeling}

The two-site model was used to simulate the concentrations eluting from the columns. A series of simulations was conducted to determine the need for fitting various parameters: (1) assuming equilibrium (i.e., $F_{i}=1$ ), (2) fitting $F_{i}$ and calculating the rate $k_{i}$ using Eq 8 and coefficients from Bouchard (1998), (3) fitting $F_{i}$ and $k_{i}$, and (4) fitting $F_{i}, k_{i}$, and the two parameters required to calculate $K_{i}$ (i.e., $K_{i w}$ and $\alpha \sigma_{i}$ ). The model fits were very sensitive to the steep gradients during the flow of $\mathrm{MeOH}$ into and out of the column; thus, we did not include concentrations during the first PV, or after PV 4.8 in $\mathrm{C} 4$, in the data used for fitting.

When using an equilibrium only assumption, the model predicted the concentrations dropping much too fast and did not predict increased concentrations during the stop flows. Using calculated rate constants and fitting $F_{i}$, we saw limited improvement to the model fits compared to the equilibrium assumption, indicating that the rates were likely too high. When fitting both $k_{i}$ and $F_{i}$, we were able to better capture the tails and predict the increase after the stops; however, the peak concentrations were often underestimated. When fitting the four parameters, $F_{i}, k_{i}, K_{i w}$ and $\alpha \sigma_{i}$, we were better able to capture both the tails and predict the peak concentrations. The fitted parameter values along with the $L_{2}$ norm of the error are provided in Table 8.

The parameters in Table 8 data were used to model the effluent concentrations and are compared to the data in Fig 7, 8, and 9 for columns C2, C3 and C4, respectively. In general, the model provided a good fit to the experimental data. For $\mathrm{C} 2$, the concentrations were well predicted throughout the experiment for most chemicals and the average $L_{2}$ norm of the error was 0.99 . For $\mathrm{C} 3$, the average $L_{2}$ norm of the error was higher at 1.6. This experiment included a stop flow. For most of the PAHs, the model consistently was unable to fit the concentrations immediately prior to the stop in flow. The model also tended to predict higher concentrations in the effluent after the stop flow. In $\mathrm{C} 4$, the average $L_{2}$ norm was 1.08, similar to $\mathrm{C} 2$; however, considerably better fits were obtained for the lower MW PAHs. The average $L_{2}$ error was 0.63 for the two-ring PAHs and 1.45 for the five ring PAHs.

In Fig 10a and 10b, the fitted values for $K_{i w}$ and $\alpha \sigma_{i}$ are plotted for each of the columns and compared to the values from the batch experiments. For most of the two-, three-, and fourringed compounds, there was excellent agreement between the fitted values across the columns. For these compounds, the fitted values of $K_{i w}$ tended to be lower than the batch data, while the values of $\alpha \sigma_{i}$ tended to be higher. This trend was less evident for the fiveand six-ringed compounds, for which there was a lot more scatter in the data.

In Fig 10c and 10d, the fitted values for $F_{i}$ and $k_{i}$ are plotted. $F_{i}$ did not show any trend across chemicals but varied between the columns. The average value for $F_{i}$ was 0.071 in $\mathrm{C} 2$, 0.62 in $\mathrm{C} 3$, and 0.42 in $\mathrm{C} 4$. For the rate constant, the values tended to be higher for the three- and four-ringed PAHs; however, there was a lot of scatter in the data. An additional set of model fits were conducted where $F_{i}$ was set to a constant value for a given column using these average values. It was thought that fixing $F_{i}$ might be a mechanistically reasonable assumption. For fits based upon this restriction, although the individual values of $k_{i}$ varied, the overall trends in the data remained the same compared to the four parameter fits reported above.

\section{Discussion}

In this work, we investigated the remediation of field-contaminated media from a FMGP using cosolvent flushing. These experiments were conducted in systems with varying hydrodynamic characteristics. Specifically, the longest column, C2, had much greater 
dispersivity than the smaller columns, which is likely due to increased heterogeneity in the column packing. The increase in dispersivity with length scale is a well-documented phenomena that is generally attributed to increased heterogeneity (Gelhar et al., 1992). We found that removal was less efficient in the largest column (e.g., $110 \mathrm{~cm}$ ), such that more PVs were needed for the same amount of PAH removed. This result is consistent with other studies that have shown recovery efficiency decreases with length scale and heterogeneity (Soga et al., 2004). The higher linear velocity in C2 may also have contributed to less efficient removal given the importance of rate effects. Though the $110 \mathrm{~cm}$ column is still relatively small when compared to field scale, the results from this column provide a better indication of the efficiency of cosolvent flushing in field-scale systems.

Results from other studies have suggested that using a high $f_{c}$ would eliminate rate and chromatographic effects (Brusseau et al., 1991; Augustijn et al., 1994; Bouchard, 1998). The results here suggest that this is not the case for field contaminated soil. When flow was temporarily halted, we found rate effects to be present in all the small columns. NAP in particular appeared to be highly rate-limited, despite being the most soluble PAH considered. Other than NAP, we also found that the most soluble PAHs tended to elute before the least soluble, higher MW PAHs. The chromatographic effects were most prominent in the largest column.

Experimental data were fit using a two-site model. In general, the model provided a reasonable fit to the experimental data. As reported previously, the model was found to be extremely sensitive to the equilibrium partition coefficient, $K_{i}$, (Bouchard, 1998), and even small changes in this parameter could provide significant improvements to the model fits. The values for $K_{i}$ were estimated as a function of $K_{i w}$ and $\alpha \sigma_{i}$. During the model fitting, the values for $K_{w i}$ and $\alpha \sigma_{i}$ were constrained by the $99 \%$ confidence interval of the predicted values based on the batch tests. In batch tests, many of the high-MW PAHs were below the quantification limit for low values of $f_{c}$. These compounds also tended to have the most error in the model fits. Using improved analytical methods for batch tests may provide better data, which would further improve model fits.

Values for $K_{i}$ are also a function of $f_{c}$, which varied over the course of the experiment as $\mathrm{MeOH}$ displaced the water in the column. Following Augustijn et al. (1994), we initially used the dispersivity from a conservative tracer to model the cosolvent breakthrough curve. In column C4, we measured density of the effluent and found evidence of sharpening of the cosolvent front, consistent with other miscible displacement studies (Harmon et al., 1999). In addition to sharpening of the $\mathrm{MeOH}$ front, we also found that the $\mathrm{MeOH}$ breakthrough curve deviated from the advection-dispersion equation. It is not clear if this deviation is caused by instabilities that developed despite the stable displacement (i.e., downward flush of cosolvent) or due to interactions between $\mathrm{MeOH}$ and the soil organic matter. In past studies, $\mathrm{MeOH}$ has been shown to impact the sorptive properties of natural organic matter (Bouchard, 2002). More work is need to understand how MeOH may be interacting with the organic phase in FMGP soils.

Raoult's Law can be used to account for compositional changes during tar dissolution (Peters et al., 1999). Estimating equilibrium concentrations according to Raoult's Law would require an estimate of the amount of tar mass remaining on the soil, which would be difficult to ascertain given the degree of aging. Rather than estimating equilibrium concentrations, we based these concentrations on measured values for $K_{i}$ determined from batch studies. Given Raoult's Law, $K_{i}$ may vary with composition when the average molecular weight changes. Because the concentrations of PAH are a small fraction of the total organic matter, we do not expect the average molecular weight to vary greatly during cosolvent flushing. 
Parameter values for $F_{i}$ and $k_{i}$ were determined by fitting the model to experimental data. In previous studies, $F_{i}$ was shown to decrease to near zero as $f_{c}$ increased (Brusseau et al., 1991; Augustijn et al., 1994). In this work we used a very high $f_{c}$, and yet the best fit value for $F_{i}$ was over 0.4 for most of the chemicals in the smaller columns. Similar to other studies, we did not see any trend for $F_{i}$ between chemicals; however, we did see a difference in the $F_{i}$ between systems with varying hydrodynamic characteristics. Specifically, we found that $F_{i}$ decreased as the dispersion coefficient increased.

Unlike past studies, we did not find a strong correlation between $k_{i}$ and $K_{i}$, according to Eq. 8. In Fig 11, we compare rate constants determined for the aged-FMGP soil to values calculated using Eq. 8. The rate constants for the aged soil were approximately two to three orders of magnitude less than values based on artificially contaminated sand. Other studies have found these relationships provided a good fit to experimental data (Augustijn et al., 1994); however, previous studies replicated the experimental set-up used to derive the $k_{i}$ and $K_{i}$ relationship, using single-solute systems, $\sim 5$-cm columns, and relatively high flow rates. As noted by Augustijn et al. (1994), high flow rates were necessary to induce rate effects. They also noted that rate effects diminished with higher $f_{c}$. In this study, flow rates were much slower and we used a higher $f_{c}$, yet we still observed rate effects in all our column experiments.

Although the model provided a reasonable fit to the data, the fitted parameters do not necessary reflect the physical phenomena of the system. Others have shown that as $f_{c}$ increases, the value for $k_{i}$ increases and the value for $F_{i}$ decreases (Brusseau et al., 1991; Augustijn et al., 1994). This is some-what counterintuitive. As $k_{i}$ increases, the effluent concentrations approach the model prediction of the equilibrium only assumption, $F_{i}=1$. We found that using values for $k_{i}$ predicted from Eq. 8 resulted in the model being completely insensitive to the chosen value for $F_{i}$. We believe better modeling approaches are needed in which the model parameters have a well-defined physical meaning.

It is also important to recognize that this model is a simplification of a very complex system, especially in terms of the nature of the organic phase. In our analysis, we only monitored PAH concentrations, but we would expect other compounds to also be removed during flushing. In other words, we not only have desorption of PAHs from the organic phase, but the organic phase is itself undergoing some degree of dissolution. We do not know how the other components of the organic phase (e.g., polar compounds and asphaltenes) are impacted by cosolvent flushing. Improvements can be gained from a better understanding of this dissolution process. Future models may also be improved by explicit consideration of the flow equation to account for the non-ideal flow behavior of $\mathrm{MeOH}$ during cosolvent flushing.

\section{Conclusion}

These results illustrate the importance of using field samples in order to understand the complexity of contaminant behavior in environmental systems. We found previous conclusions and relationships developed based on artificially contaminated sands did not translate to the field-contaminated soil used in this study. Existing relationships to predict mass transfer rates were incapable of simulating the desorption from field contaminated media. Though we significantly reduced the chromatographic effect, we still found that compounds were removed over varying time scales, particularly in the largest column. In evaluating cosolvent flushing, it is extremely important to consider compounds on an individual basis, particularly for higher MW compounds that can drive human health risk. Interestingly, NAP was also extremely recalcitrant despite being the most soluble PAH, which was also contrary to results based on artificially contaminated media. 
Cosolvent flushing has the potential to remove large quantities of contaminant mass with a minimum number of PVs, compared to water flushing alone. In the largest column studied, $10 \mathrm{PVs}$ of cosolvent removed almost $90 \%$ of PAH mass while > 100 PVs of water during the equilibration period had little effect on the concentrations of PAHs in the soil. For a particular site, the appropriateness of cosolvent flushing will be dependent on a number of factors including site heterogeneity and the particular remediation goal. In addition, $10 \mathrm{PVs}$ of flushing solution on a field scale can still be prohibitively large. Regardless, removing all of the contaminant mass is likely to be an unachievable goal even when using a high cosolvent fraction. Batch and column studies can be used to understand how a particular media will respond to a given remediation technology, such as cosolvent flushing. Improvements to models will aid our ability to evaluate the effectiveness of cosolvent flushing over temporal and spatial scales and provide reasonable estimates of the number of PVs required for a given remediation goal. Of particular importance is the consideration of density and viscosity variations that will impact the effectiveness of flushing at the field scale.

\section{Acknowledgments}

This work was supported by National Institute of Environmental Health Sciences grant 5 P42 ES05948.

\section{References}

ASTM. Annual Book of ASTM Standards. Vol. 4.08. American Society for Testing and Materials; Philadelphia, PA: 1999a. Standard test method for laboratory determination of water (moisture) content of soil and rock by mass, D2216-98; p. 190-194.

ASTM. Annual Book of ASTM Standards. Vol. 4.08. American Society for Testing and Materials; Philadelphia, PA: 1999b. Standard test method for particle-size analysis of soils, D422-63; p. 10-17.

ASTM. Annual Book of ASTM Standards. Vol. 4.08. American Society for Testing and Materials; Philadelphia, PA: 1999c. Standard test method for specific gravity of soil solids by water pycnometer, D854-92.

ASTM. Annual Book of ASTM Standards. Vol. 4.08. American Society for Testing and Materials; Philadelphia, PA: 2001. Standard test method for pH of soils, D4972-01.

Augustijn DCM, Jessup RE, Rao PSC, Wood AL. Remediation of contaminated soils by solvent flushing. Journal of Environmental Engineering. 1994; 120 (1):42-57.

Barranco FT, Dawson HE. Influence of aqueous ph on the interfacial properties of coal tar. Environmental Science \& Technology. 1999; 33 (10):1598-1603.

Birak PS, Miller CT. Dense non-aqueous phase liquids at former manufactured gas plants: Challenges to modeling and remediation. Journal of Contaminant Hydrology. 2009; 105 (3-4):81-172. [PubMed: 19176266]

Bouchard DC. Sorption kinetics of PAHs in methanol-water systems. Journal of Contaminant Hydrology. 1998; 34 (1-2):107-120.

Bouchard DC. Cosolvent effects on sorption isotherm linearity. Journal of Contaminant Hydrology. 2002; 56 (3-4):159-174. [PubMed: 12102316]

Brusseau ML, Wood AL, Rao PSC. Influence of organic cosolvents on the sorption kinetics of hydrophobic organic chemicals. Environmental Science \& Technology. 1991; 25 (5):903-910.

Chen CS, Rao PSC, Delfino JJ. Oxygenated fuel induced cosolvent effects on the dissolution of polynuclear aromatic hydrocarbons from contaminated soil. Chemosphere. 2005; 60 (11):15721582. [PubMed: 16083763]

Farthing MW, Miller CT. A comparison of high-resolution, finite-volume, adaptive-stencil schemes for simulating advective-dispersive transport. Advances in Water Resources. 2000; 24 (1):29-48.

Gelhar LW, Welty C, Rehfeldt KR. A critical review of data on field-scale dispersion in aquifers. Water Resources Research. 1992; 28 (7):1955-1974. 
Gonzalez B, Calvar N, Gomez E, Dominguez A. Density, dynamic viscosity, and derived properties of binary mixtures of methanol or ethanol with water, ethyl acetate, and methyl acetate at $\mathrm{T}=(293.15$, 298.15, and 303.15)K. The Journal of Chemical Thermodynamics. 2007; 39 (12):1578-1588.

Haeseler F, Blanchet D, Druelle V, Werner P, Vandecasteele JP. Analytical characterization of contaminated soils from former manufactured gas plants. Environmental Science \& Technology. 1999; 33 (6):825-830.

Harden SL, Chapman MJ, Harned DA. Characterization of groundwater quality based on a regional geologic setting in the Piedmont and Blue Ridge Physiographic Provinces, North Carolina. Tech rep, US Geological Survey. 2009

Harmon TC, Kim TJ, Dela Barre BK, Chrysikopoulos CV. Cosolvent-water displacement in onedimensional soil column. Journal of Environmental Engineering-Asce. 1999; 125 (1):87-91.

Kilbane JJ. Extractability and subsequent biodegradation of PAHs from contaminated soil. Water Air and Soil Pollution. 1998; 104 (3-4):285-304.

Liu LH, Endo S, Eberhardt C, Grathwohl P, Schmidt TC. Partition behavior of polycyclic aromatic hydrocarbons between aged coal tar and water. Environmental Toxicology and Chemistry. 2009; 28 (8):1578-1584. [PubMed: 19309178]

Lukasewycz MT, Burkhard LP. Complete elimination of carbonates: A critical step in the accurate measurement of organic and black carbon in sediments. Environmental Toxicology and Chemistry. 2005; 24 (9):2218-2221. [PubMed: 16193748]

Luthy RG, Dzombak DA, Peters CA, Roy SB, Ramaswami A, Nakles DV, Nott BR. Remediating tarcontaminated soils at manufactured gas plant sites. Environmental Science \& Technology. 1994; 28 (6):266A-276A.

Peters CA, Knightes CD, Brown DG. Long-term composition dynamics of PAH-containing NAPLs and implications for risk assessment. Environmental Science \& Technology. 1999; 33 (24):4499_ 4507.

Peters CA, Mukherji S, Knightes CD, Weber JWJ. Phase stability of multicomponent NAPLs containing PAHs. Environmental Science \& Technology. 1997; 31 (9):2540-2546.

Powers SE, Anckner WH, Seacord TF. Wettability of NAPL-contaminated sands. Journal of Environmental Engineering - ASCE. 1996; 122 (10):889-896.

Rao PS, Lee LS, Pinal R. Cosolvency and sorption of hydrophobic organic chemicals. Environmental Science \& Technology. 1990; 24 (5):647-654.

Richardson SD, Lebron BL, Miller CT, Aitken MD. Recovery of phenanthrene-degrading bacteria after simulated in situ persulfate oxidation in contaminated soil. Environmental Science \& Technology. 2011; 45 (2):719-725. [PubMed: 21162560]

Soga K, Page JWE, Illangasekare TH. A review of NAPL source zone remediation efficiency and the mass flux approach. Journal of Hazardous Materials. 2004; 110 (1-3):13-27. [PubMed: 15177723]

U.S. EPA. Tech Rep EPA/600/R-93/089. U.S. Environmental Protection Agency; Washington, D. C: 1993. Provisional guidance for quantitative risk assessment of polycyclic aromatic hydrocarbons.

U.S. EPA. Tech Rep EPA/542/R-04/015. U.S. Environmental Protection Agency; Cincinnati, OH: 2004. Cleaning up the nation's waste sites: Markets and technology trends.

Wood AL, Bouchard DC, Brusseau ML, Rao PS. Cosolvent effects on sorption and mobility of organic contaminants in soils. Chemosphere. 1990; 21 (4-5):575-587.

Yalkowsky, SH. Solubility and solubilization of nonelectrolytes; solubilization of drugs by cosolvents. In: Yalkowsky, SH., editor. Techniques of Solubilization of Drugs. Marcel Dekker, Inc; New York: 1981. p. 1-14.p. 91-134. 


\section{Highlights}

- Complex, aged, field-contaminated tar material investigated for cosolvent remediation of PAH contaminants

- Standard log-linear model described equilibrium between the solid and the cosolvent as a function of methanol volume fraction

- Chromatographic effects were observed as a function of solubility of the PAH compound

- Desorption rate modeling showed rate coefficients were two to three orders of magnitude smaller than previously reported for artificially contaminated materials 
(a) PHE

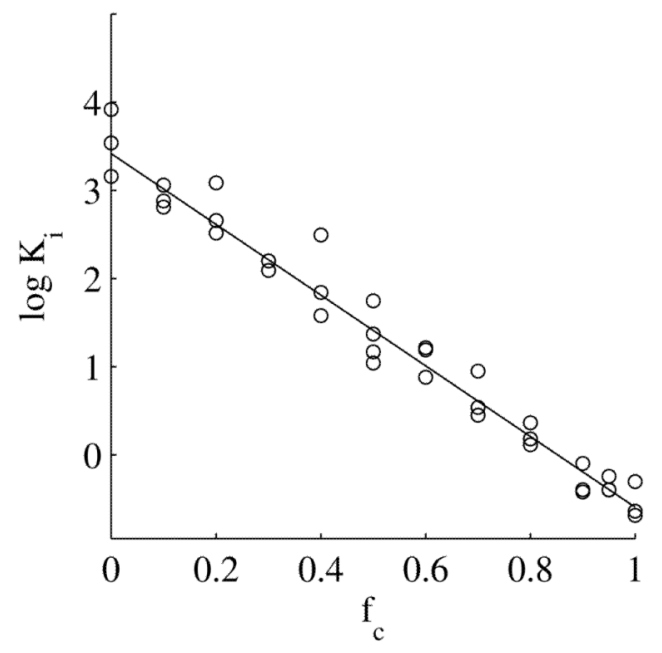

(b) $\mathrm{BaP}$

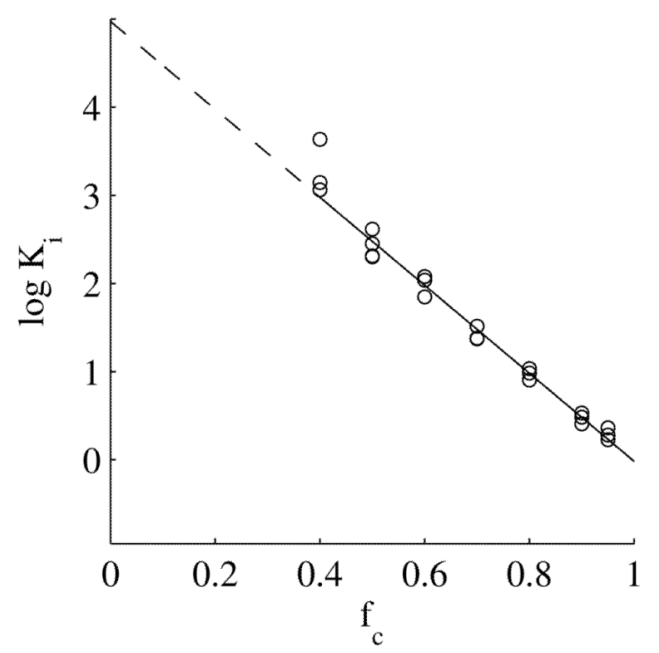

\section{Fig. 1.}

Least-square fits to batch data using the log-linear model (Eq. 2) for PHE and BaP. The dashed line represents values extrapolated beyond the experimental data. 
(a) Tritium, $\mathrm{D}=4,680 \mathrm{~cm}^{2} / \mathrm{d}$

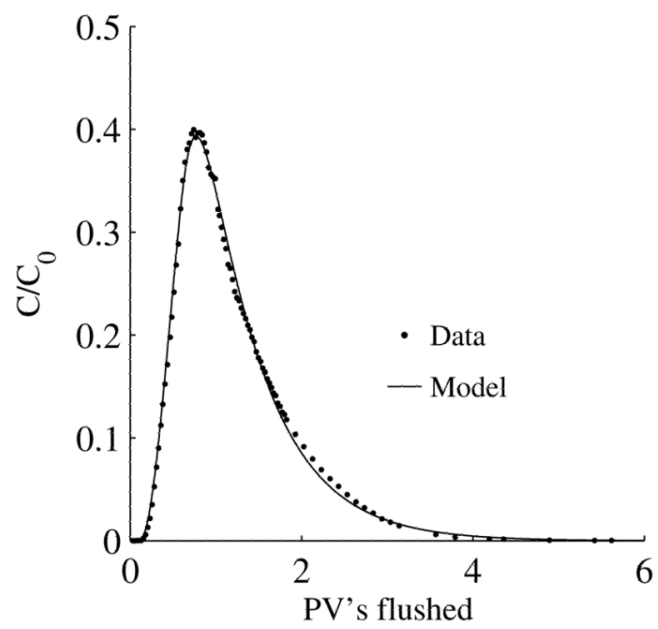

(b) $\mathrm{MeOH}, \mathrm{D}=4,680 \mathrm{~cm}^{2} / \mathrm{d}$

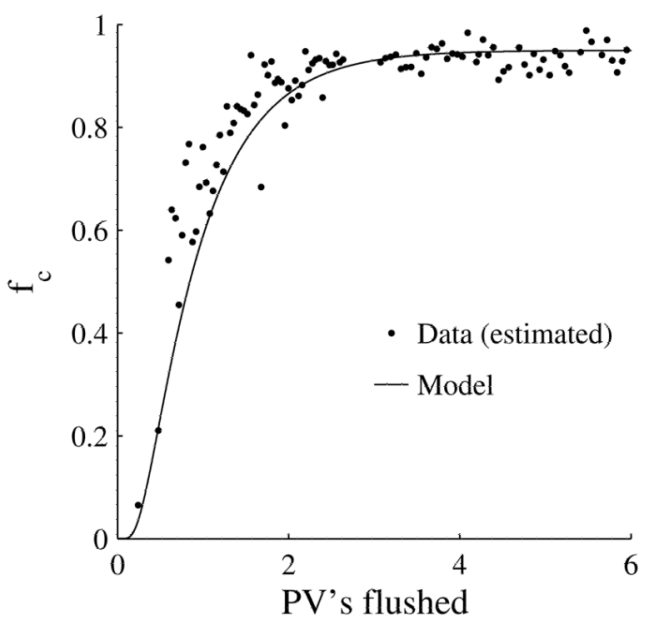

Fig. 2.

Tritium and $\mathrm{MeOH}$ concentration as a function of PVs flused in $\mathrm{C} 2(110 \mathrm{~cm})$. The dispersivity from the tracer test was used to estimate the methanol $f_{c}$. 

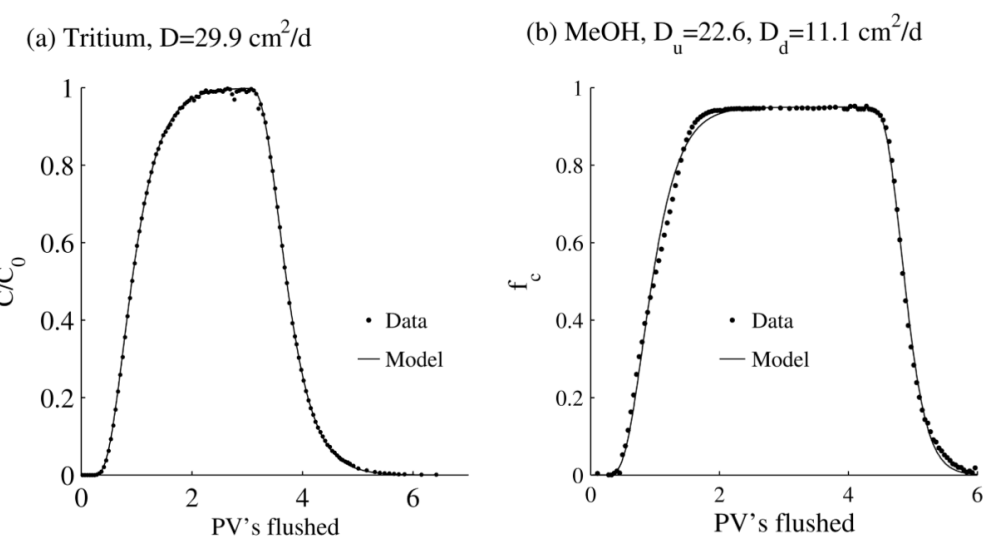

Fig. 3.

Tritium and $\mathrm{MeOH}$ concentration as a function of PVs flushed in $\mathrm{C} 4(11.9 \mathrm{~cm})$. The $\mathrm{MeOH}$ data was fit separately to determine the dispersion coefficient during the breakthrough and elution portions of the curve. 


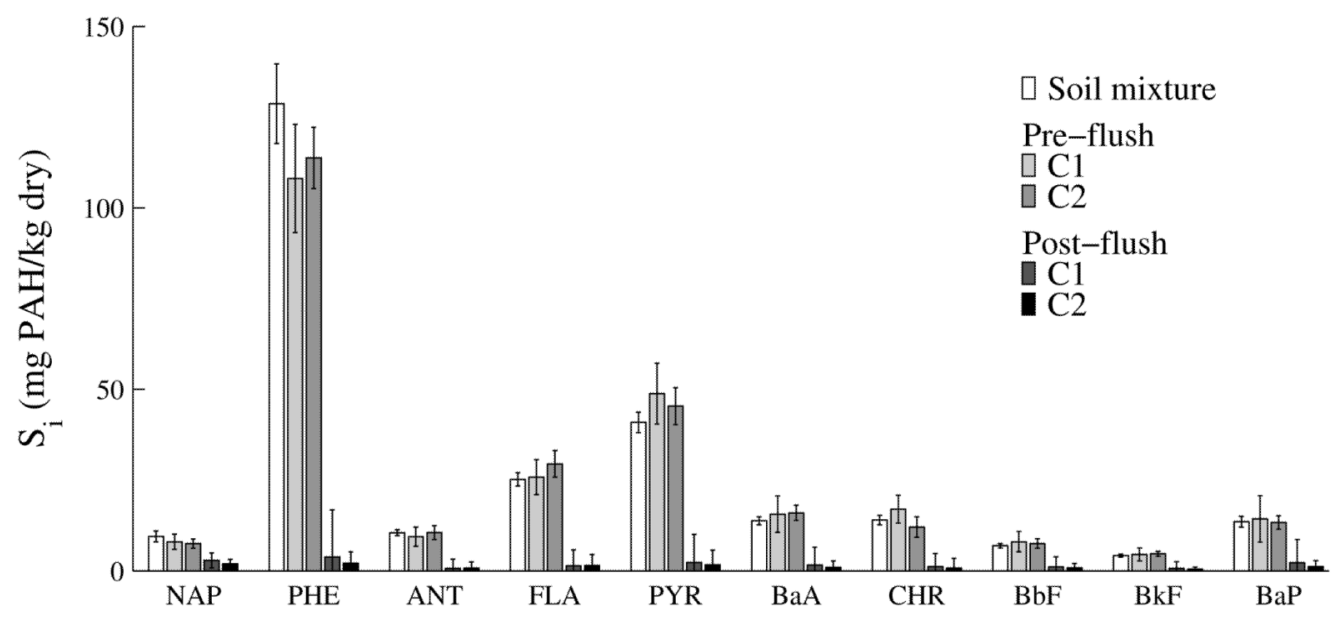

Fig. 4.

Comparison between $S_{i}$ pre- and post-flushing in columns C1 $(22.2 \mathrm{~cm})$ and C2 $(110 \mathrm{~cm})$. The pre-flushing values compare favorably to the original soil mixture. 

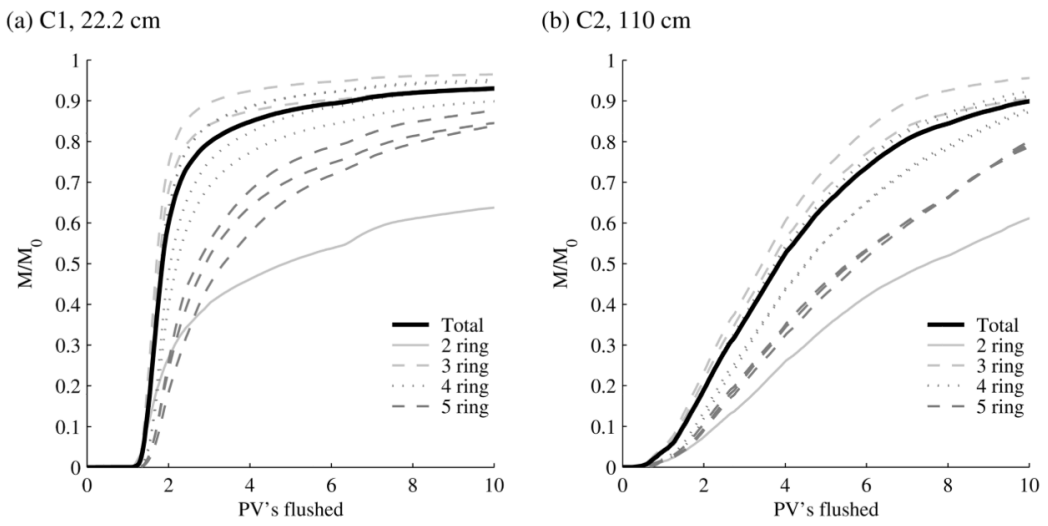

Fig. 5.

PAH mass removal as a function of PV in columns $\mathrm{C} 1$ and $\mathrm{C} 2$ after flushing $10 \mathrm{PVs}$ of cosolvent $\left(\mathrm{MeOH} f_{c}=0.95\right)$ for the 10 PAHs noted in Fig 4. 

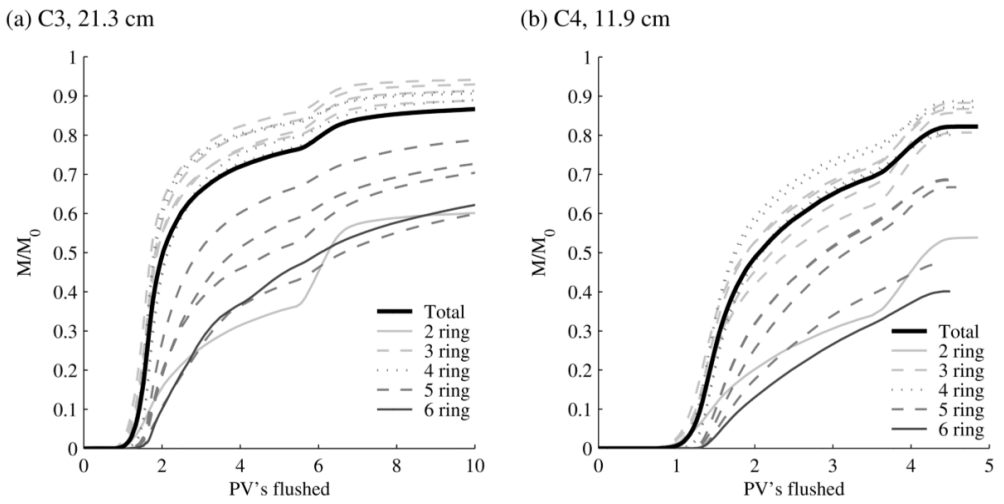

Fig. 6.

PAH mass removal as a function of PV in columns $\mathrm{C} 3$ and $\mathrm{C} 4$ during cosolvent flushing $\left(\mathrm{MeOH} f_{c}=0.95\right)$ for the 14 PAHs in Table 5. Flow was stopped at 5.6 PVs in C3 and 3.8 PVs in C4. In C4, the column was flushed with water immediately after the stop flow, while C3 was flushed with additional cosolvent. 

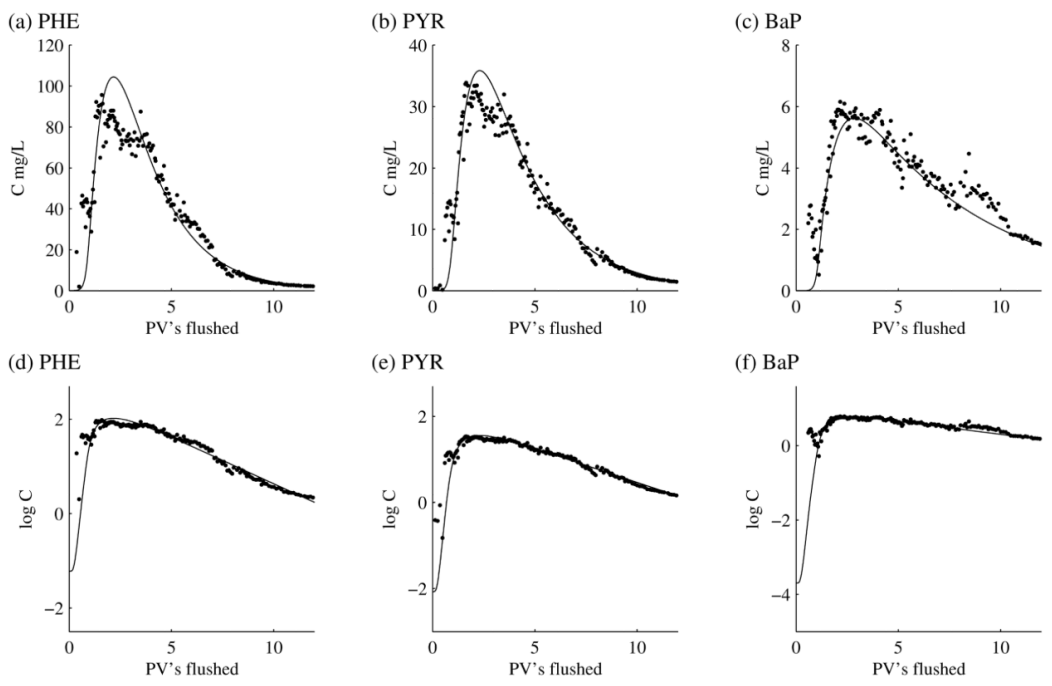

Fig. 7.

Modeled effluent concentrations compared to data from $\mathrm{C} 2$. 

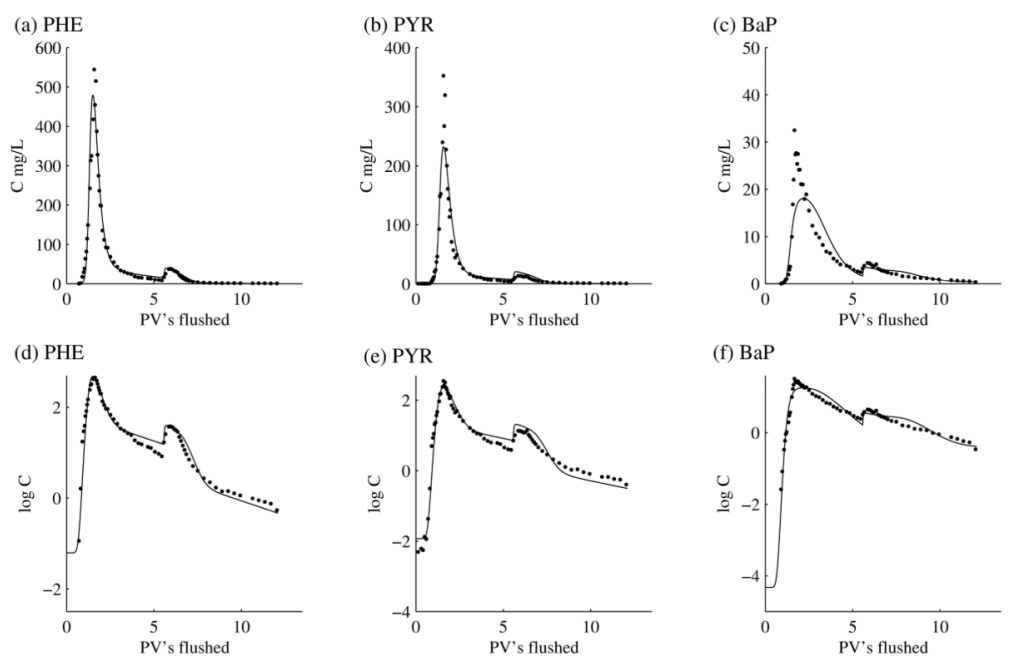

Fig. 8.

Modeled effluent concentrations compared to data from C3. 

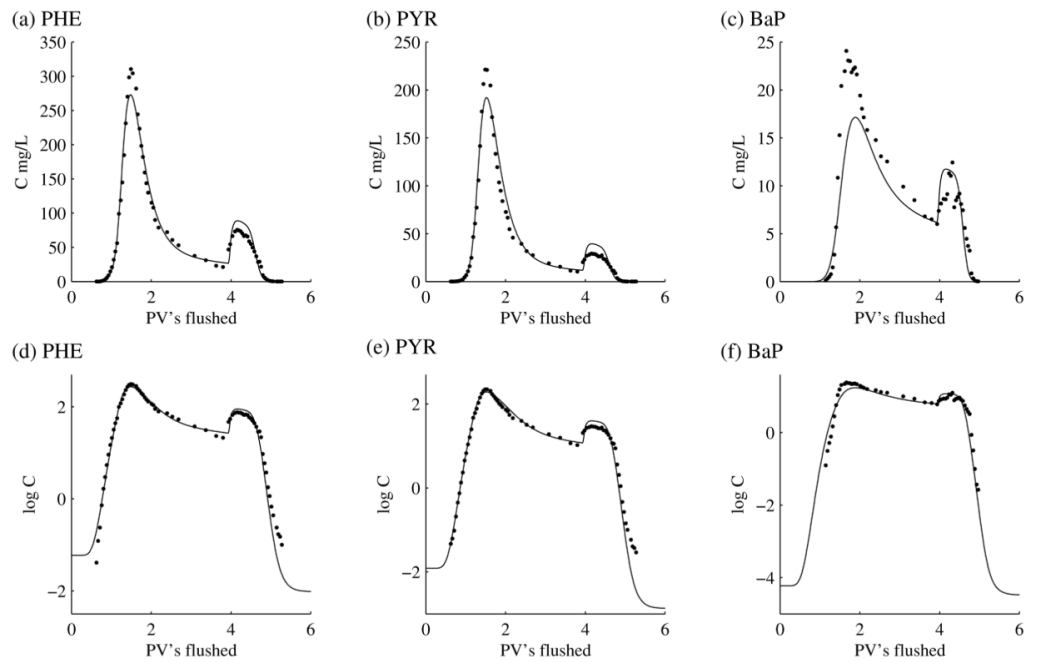

Fig. 9.

Modeled effluent concentrations compared to data from $\mathrm{C} 4$. 
(a)

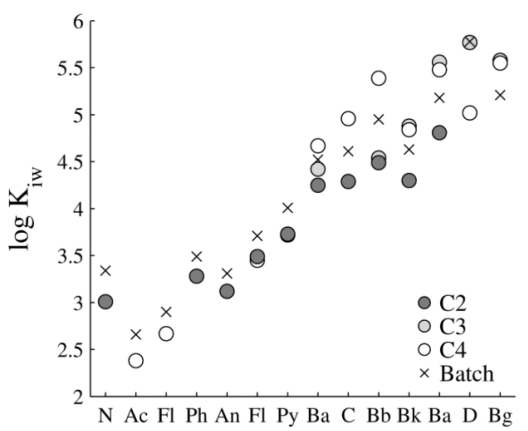

(c)

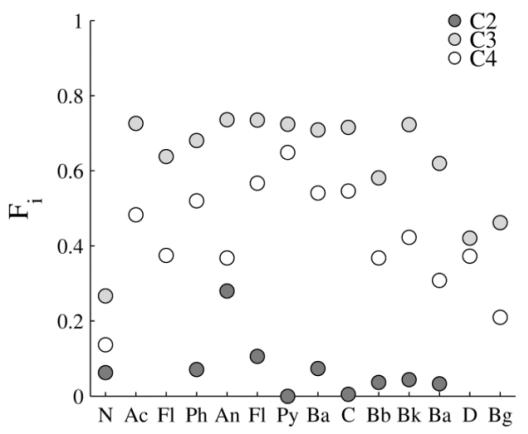

(b)

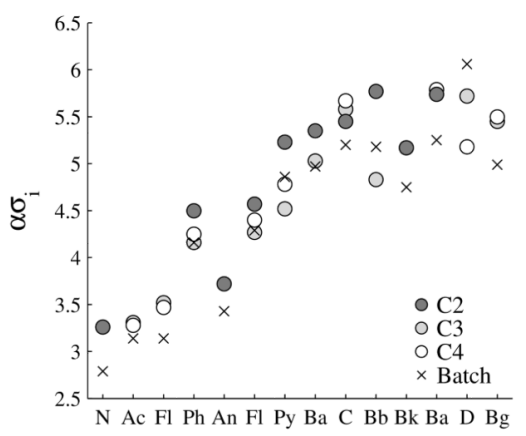

(d)

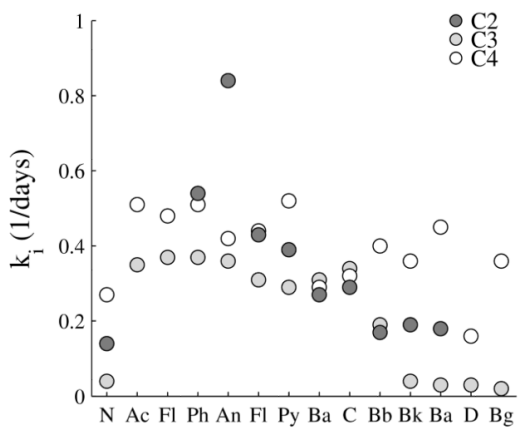

Fig. 10.

Fitted parameter values. 


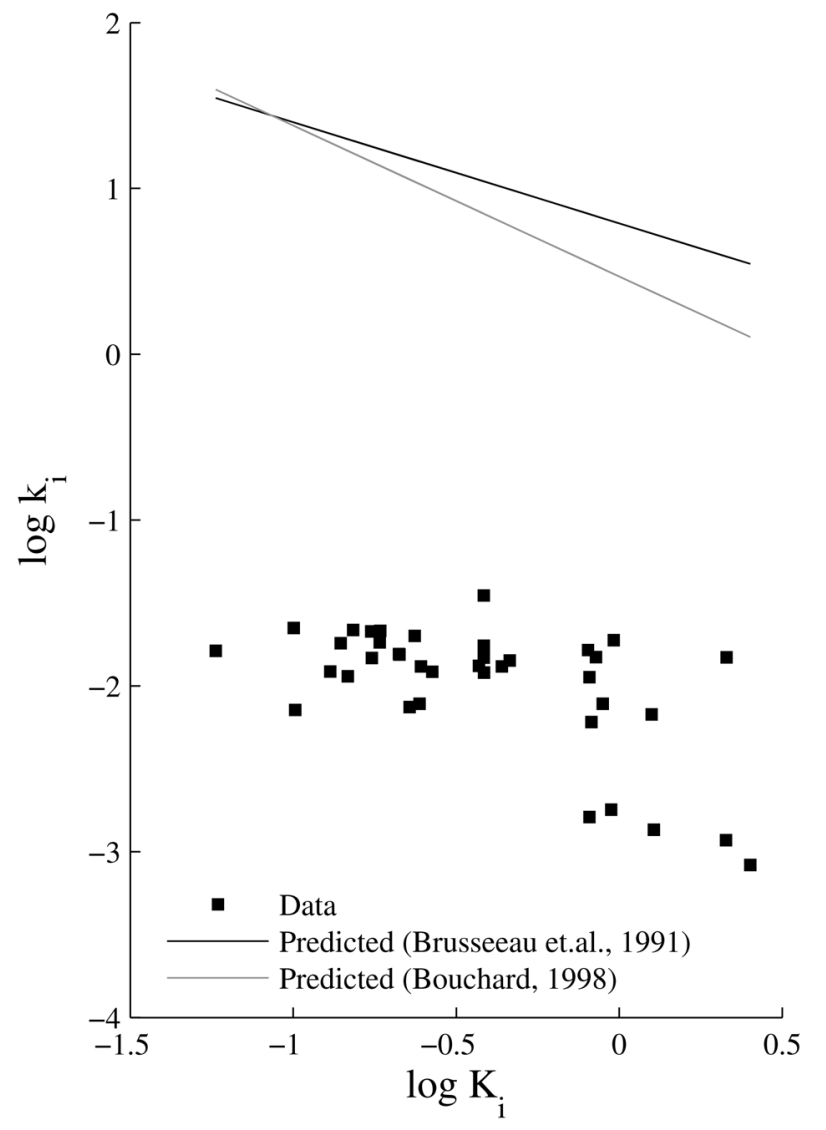

Fig. 11.

Relationship between the mass transfer rate, $\log k_{i}$, and the partition co-efficient, $\log K_{i}$ $\left(f_{c}=0.95\right)$. The $k_{i}$ data are for the FMGP soil (Table 8$)$ and were converted to units of $\mathrm{hr}^{-1}$ to compare with predicted values from Eq. 8, which were based on artificially contaminated sands. 
Table 1

Properties of the soil mixture

\begin{tabular}{|c|c|c|c|c|}
\hline Property & $\mathbf{n}$ & Mean & SD & Method \\
\hline$\%$ Sand & 1 & 82.9 & & D422-63, ASTM (1999b) \\
\hline$\%$ Silt & 1 & 13.8 & & D422-63, ASTM (1999b) \\
\hline$\%$ Clay & 1 & 3.3 & & D422-63, ASTM (1999b) \\
\hline$\%$ Moisture content & 6 & 7.50 & 0.01 & D2216-98, ASTM (1999a) \\
\hline $\mathrm{pH}$ & 3 & 7.56 & 0.11 & D4972-01, ASTM (2001) \\
\hline Particle density $\left(\mathrm{g} / \mathrm{cm}^{3}\right)$ & 3 & 2.62 & 0.04 & D854-92, ASTM (1999c) \\
\hline$\%$ Inorganic carbon (IC) ${ }^{a}$ & 6 & 7.0 & 1.6 & Lukasewycz and Burkhard (2005) \\
\hline$\%$ Organic carbon $(\mathrm{OC})^{a}$ & 3 & 8.3 & 1.2 & Lukasewycz and Burkhard (2005) \\
\hline$\%$ Total extractable organic matter (TEOM) ${ }^{a}$ & 15 & 0.64 & 0.10 & See $\$ 2.2$ \\
\hline
\end{tabular}

$a_{\text {Dry mass basis. }}$ 
Table 2

Density as a function of $\omega$ and $f_{c}$ at $22^{\circ} \mathrm{C}(\mathrm{n}=3)$

\begin{tabular}{llll}
\hline & & $\boldsymbol{\rho}(\mathrm{g} / \mathrm{ml})$ \\
$\boldsymbol{\omega}$ & $\boldsymbol{f}_{\boldsymbol{c}}$ & Mean & Stdev \\
\hline 0.000 & 0.000 & 0.9977 & 0.0002 \\
0.040 & 0.050 & 0.9906 & 0.0001 \\
0.080 & 0.101 & 0.9842 & 0.0004 \\
0.165 & 0.204 & 0.9710 & 0.0002 \\
0.253 & 0.308 & 0.9578 & 0.0003 \\
0.345 & 0.414 & 0.9426 & 0.0002 \\
0.441 & 0.519 & 0.9254 & 0.0003 \\
0.441 & 0.519 & 0.9254 & 0.0003 \\
0.543 & 0.624 & 0.9052 & 0.0003 \\
0.648 & 0.726 & 0.8819 & 0.0001 \\
0.759 & 0.826 & 0.8552 & 0.0001 \\
0.877 & 0.919 & 0.8244 & 0.0001 \\
0.938 & 0.964 & 0.8074 & 0.0001 \\
1.000 & 1.000 & 0.7898 & 0.0003 \\
\hline
\end{tabular}


Table 3

Column apparatus and conditions during cosolvent flushing experiments

\begin{tabular}{lllll}
\hline & C1 & C2 & C3 & C4 \\
\hline Mass of soil mixture $(\mathrm{kg})$ & 0.160 & 13.4 & 0.170 & 0.082 \\
Total height $(\mathrm{cm})$ & 22.2 & 110 & 21.3 & 11.9 \\
Inner diameter $(\mathrm{cm})$ & 2.5 & 9.83 & 2.5 & 2.5 \\
Porosity, $\theta$ & 0.401 & 0.417 & 0.382 & 0.397 \\
Linear pore velocity, $v(\mathrm{~cm} /$ day $)$ & 24.2 & 109 & 25.5 & 24.7 \\
Mean residence time (day) & 0.92 & 1.0 & 0.84 & 0.48 \\
Hydrodynamic dispersion coefficient, $D\left(\mathrm{~cm}^{2} /\right.$ day) & - & 4,680 & 22.3 & 29.9 \\
Peclet number & - & 2.56 & 24.4 & 9.83 \\
\hline
\end{tabular}


Table 4

Comparison of cosolvent flushing experiments

\begin{tabular}{lllll}
\hline & C1 & C2 & C3 & C4 \\
\hline Total PVs Flushed & 10.6 & 11.9 & 12.1 & 3.8 \\
PVs Prior to Stop & 6.2 & - & 5.6 & 3.8 \\
Stop Time & $2 \mathrm{~d}$ & - & $2 \mathrm{wk}$ & $4 \mathrm{~d}$ \\
\hline
\end{tabular}


Table 5

PAH analysis of the soil mixture $(n=33)$

\begin{tabular}{|c|c|c|c|c|}
\hline \multirow[b]{2}{*}{ Chemical } & \multirow[b]{2}{*}{ Abbreviation } & \multirow[b]{2}{*}{ MW } & \multicolumn{2}{|c|}{$S_{i}(\mathrm{mg}$ PAH/kg dry $)$} \\
\hline & & & $\operatorname{Mean}^{a}$ & Range \\
\hline \multicolumn{5}{|l|}{2 Rings } \\
\hline Naphthalene & NAP & 128 & $9.5 \pm 1.5$ & $4.5-25.6$ \\
\hline \multicolumn{5}{|l|}{3 Rings } \\
\hline Acenaphthene & $\mathrm{ACE}$ & 152 & $11.9 \pm 1.0$ & $8.4-20.1$ \\
\hline Fluorene & FLE & 166 & $9.5 \pm 0.7$ & $6.6-15.8$ \\
\hline Phenanthrene & PHE & 178 & $129 \pm 11$ & $73-254$ \\
\hline Anthracene & ANT & 178 & $10.5 \pm 0.8$ & $6.0-17.4$ \\
\hline \multicolumn{5}{|l|}{4 Rings } \\
\hline Fluoranthene & FLA & 202 & $25.2 \pm 1.8$ & $15.6-39.5$ \\
\hline Pyrene & PYR & 202 & $40.9 \pm 2.8$ & $25.5-65.5$ \\
\hline Benzo[a]anthracene & $\mathrm{BaA}$ & 228 & $13.8 \pm 1.1$ & $8.9-22.3$ \\
\hline Chrysene & CHR & 228 & $14.0 \pm 1.3$ & $9.1-22.7$ \\
\hline \multicolumn{5}{|l|}{5 Rings } \\
\hline Benzo[b]fluoranthene & $\mathrm{BbF}$ & 252 & $6.9 \pm 0.6$ & $4.1-13.2$ \\
\hline Benzo[k]fluoranthene & $\mathrm{BkF}$ & 252 & $4.2 \pm 0.4$ & $2.3-8.3$ \\
\hline Benzo[a]pyrene & $\mathrm{BaP}$ & 252 & $13.5 \pm 1.5$ & $8.1-28.5$ \\
\hline Dibenzo[ah]anthracene & DBA & 278 & $1.0 \pm 0.2$ & $0.6-3.1$ \\
\hline \multicolumn{5}{|l|}{6 Rings } \\
\hline Benzo[ghi]perylene & $\mathrm{BgP}$ & 276 & $5.3 \pm 0.6$ & $2.8-10.9$ \\
\hline Total PAHs & & & $295 \pm 12$ & \\
\hline
\end{tabular}

${ }^{a}$ Confidence interval is the 95 th percentile around the mean. 
Table 6

Fractionation of TEOM from field soil, $n=3$

\begin{tabular}{lrcc}
\hline Fraction & Mean & SD & RSD \\
\hline Asphaltenes & 25.4 & 0.7 & 0.027 \\
Polar compounds & 20.8 & 1.9 & 0.089 \\
Neutral compounds & 45.0 & 1.4 & 0.030 \\
\hline
\end{tabular}




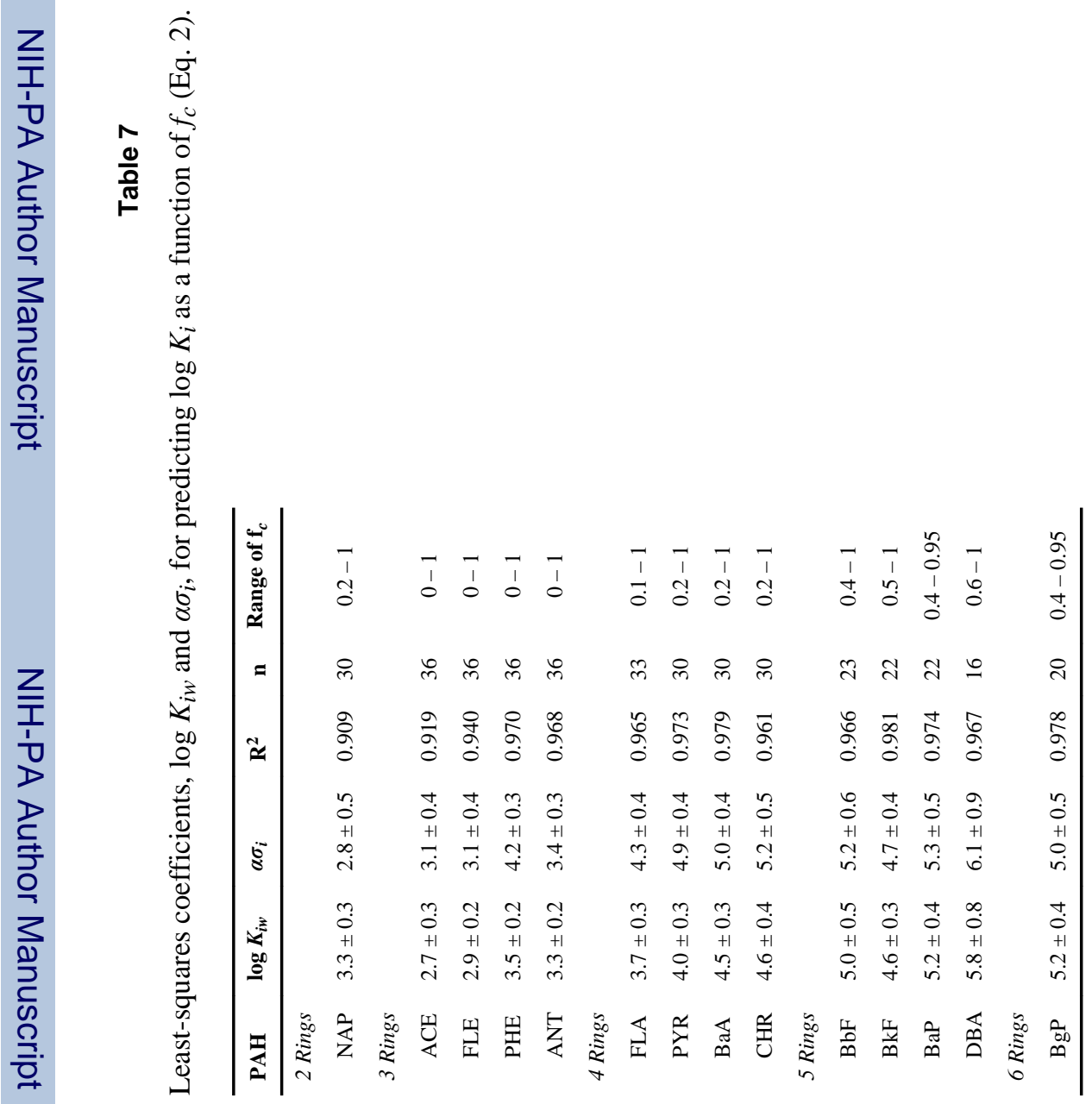


